\title{
La raison interculturelle et les conditions préliminaires de l'argumentation
}

Intercultural Reason and the Preliminary Conditions of Argumentation

\section{Christopher W. Tindale}

Translator. Ruth Amossy

\section{(2) OpenEdition Journals}

\section{Electronic version}

URL: http://journals.openedition.org/aad/4477

DOI: $10.4000 /$ aad. 4477

ISSN: 1565-8961

\section{Publisher}

Université de Tel-Aviv

\section{Electronic reference}

Christopher W. Tindale, "La raison interculturelle et les conditions préliminaires de l'argumentation », Argumentation et Analyse du Discours [Online], 25 | 2020, Online since 15 October 2020, connection on 17 October 2020. URL : http://journals.openedition.org/aad/4477 ; DOI : https://doi.org/10.4000/aad. 4477

This text was automatically generated on 17 October 2020 .

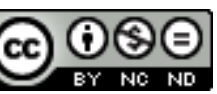

Argumentation \& analyse du discours est mis à disposition selon les termes de la licence Creative Commons Attribution - Pas d'Utilisation Commerciale - Pas de Modification 4.0 International. 


\title{
La raison interculturelle et les conditions préliminaires de l'argumentation
}

\author{
Intercultural Reason and the Preliminary Conditions of Argumentation
}

\author{
Christopher W. Tindale
}

Translation : Ruth Amossy

L'emprise du familier nous appauvrit tous

(Clifford Geertz)

\section{Les limites de la compréhension}

1 Comme les humains ont tendance à se lover dans leur cocon - un état qui les définit et assure leur stabilité - l'expérience de l'altérité s'avère abrupte et souvent perturbante. Nous le voyons avec une particulière acuité dans nos confrontations avec ceux qui raisonnent différemment de nous, ceux que nous qualifions hâtivement d'« irrationnels » parce qu'ils ne répondent pas aux critères de ce que nous considérons comme rationnel. En portant une attention plus soutenue à ce qu'ils disent et font, nous pourrions cependant, sinon l'expliquer dans nos propres termes, du moins y déceler une rationalité interne régulée par des schémas signifiants. Ces discours répondent en effet à une exigence de consistance, et peuvent se défendre selon leurs propres critères. Le problème est que ces modalités, ces schémas signifiants ne correspondent pas aux nôtres. C'est précisément ce qui rend la conversation, la discussion et l'argumentation difficiles, sinon impossibles. Pour raisonner ensemble, nous devons avoir un fonds de significations partagées ; nos positions et nos thèses doivent être compréhensibles dans leur principe, alors que dans ce cas particulier, nous nous tenons aux frontières de la compréhension sans avoir la capacité de les franchir.

2 Les commentateurs qui traitent de cette question se divisent généralement en optimistes et pessimistes. Un pessimiste comme Stanley Fish (1999) croit que nous ne pouvons pas nous entendre parce que nous partons de prémisses totalement 
incommensurables. Contrairement à ceux qui croient que «ce que nous voyons est ce que nous savons, ce que nous croyons, ce que nous sommes, ce que nous faisons ", Fish reconfigure cette série en plaçant « croyons » en tête : ce que nous croyons est ce que nous voyons, ce que nous savons, ce que nous sommes, ce que nous faisons (Fish 1999 : 247). Il y a aussi le pessimisme que professe R. M. Hare (1970), lequel déclare abruptement que nous ne pouvons pas raisonner avec le fanatique parce que le fanatique ne raisonne pas : il n'y a donc rien à faire ${ }^{1}$.

timistes se prétendent quant à eux bien plus avancés, souvent en citant à l'appui des réussites passées et des cas de différends résolus. Certains suivent la voie tracée par Mill (1999) qui plaide pour une société où les points de vue indésirables sont tolérés parce que s'ils étaient supprimés, les points de vue « corrects » ne pourraient émerger. D'autres recommandent de passer du domaine épistémique au domaine pratique pour gérer les désaccords en termes d'attribution de valeurs subjectives aux individus, en évitant ainsi la tâche périlleuse de décider de ce qui est « vrai » ou « correct ».

Chaim Perelman et Lucie Olbrechts-Tyteca se situent dans ce continuum quelque part entre le pessimiste et l'optimiste. Les expériences de la Seconde guerre mondiale qui ont mené Perelman au projet de la nouvelle rhétorique étaient marquées par l'échec des humains à se comporter selon la justice; mais le tournant de la rhétorique et de l'argumentation promettaient selon lui de fournir les instruments nécessaires pour améliorer ce genre de situations.

5 A l'instar de Hare, Perelman et Olbrechts-Tyteca se préoccupent du fanatique, un qualificatif qui comporte déjà en soi un jugement de rejet. Très tôt dans Le traité de l'argumentation, ils introduisent cette figure dans le passage suivant :

le fanatique est celui qui, adhérant à une thèse contestée, et dont la preuve indiscutable ne peut être fournie, refuse néanmoins la possibilité de la soumettre à une libre discussion, et par conséquent, refuse les conditions préalables qui permettraient, sur ce point, l'exercice de l'argumentation (1970:82).

6 Ce passage est instructif à divers égards dans la mesure où il nous apprend quelque chose sur la conduite du fanatique (bien qu'il ne dise rien de sa nature). Mais c'est surtout la dernière clause qui est intéressante en raison de la façon dont elle introduit la question des « conditions préalables » de l'argumentation. Je voudrais suggérer qu'il $\mathrm{y}$ a là un terrain fertile à explorer : ceux qui campent sur des positions opposées, où l'absence de compréhension semble n'offrir aucune perspective de progrès, doivent être impliqués à un stade préliminaire, qui précède l'argumentation. Ce sont des conditions de cet ordre que je propose d'explorer dans cet article.

\section{Dialogues de sourds}

7 Il se peut que Perelman et Olbrechts-Tyteca se réfèrent simplement à la disponibilité à s'engager dans une discussion libre ; une disponibilité qui fait défaut au fanatique. Les énoncés qui suivent n'éclairent pas la question car ils se tournent vers le sceptique qui, contrairement au fanatique, refuse tout engagement. Mais comme le sceptique, le fanatique méconnaît " que l'argumentation vise à un choix entre des possibles » (ibid. : 83). Le fanatique, n'ayant aucune décision à prendre, évite l'acte de décider et donc le rôle qu'il joue dans l'argumentation.

8 La question dont traitent Perelman et Olbrechts-Tyteca ne correspond pas exactement à ce dont j'ai parlé plus haut, bien que les liens soient apparents. A la fin de la première 
partie de leur traité, ils se focalisent sur ceux que leur attitude situe en-dehors de l'argumentation comme activité sociale: le fanatique et le sceptique. Ils restent endehors parce qu'ils échouent à saisir la nature vitale du choix. Nous sommes invités à imaginer des figures statiques, figées dans leurs croyances et leurs attitudes. La mesure dans laquelle il s'agit là d'une description réaliste de la nature humaine est une question à examiner ailleurs. Le choix implique des alternatives, et les alternatives impliquent la possibilité du changement. L'argumentation est un levier de changement, elle lui confère ses motivations et ses moyens de réalisation ${ }^{2}$.

Dans les termes de la discussion qui figure en première partie, ceux qui font l'expérience d'un choc des rationalités ne sont pas nécessairement opposés au changement et ne l'évitent pas forcément. Cependant, ils reconnaissent seulement les changements qu'ils considèrent comme "rationnels ", et ne sont prêts à les faire qu'à leur guise. Ils sont aussi enfermés dans leur position que le fanatique et le sceptique, mais d'une façon qu'on peut considérer comme reflétant plus adéquatement l'expérience humaine. Leurs points de vue ne sont pas figés ni leurs positions statiques. Mais ils sont limités. Si nous recadrons en ces termes le fanatique (ou l'extrémiste), et leur assignons une " rationalité » différente, le problème ressort clairement. Il y a une barrière qui bloque les efforts de compréhension, et le rôle de l'argumentation s'en trouve limité.

Marc Angenot (2008) saisit ce qui est ici en jeu dans une métaphore frappante : celle du dialogue de sourds, dans lequel les deux parties parlent mais n'entendent pas ce que dit l'autre. En fait, la thèse d'Angenot est discutable dans la mesure où il insiste sur le fait que ce genre de malentendu représente la norme. Remettant en question l'un des fondements de l'histoire de la rhétorique, il soutient que nos efforts de persuasion échouent immanquablement, si bien que les humains n'argumentent pas pour se persuader mutuellement. Il voit dans les différences des codes rhétoriques la racine de ce qu'il décrit comme des fossés ou des coupures cognitives: «les humains ne comprennent pas leurs raisonnements réciproques parce que, parlant la même langue, ils n'usent pas (ou pas tout à fait, nous n'avons pas établi quel quantum de divergence suffit à bloquer un débat) du même code rhétorique.» (Angenot 2008: 15) Dans cette perspective, la rhétorique se résume à la négociation ou à la gestion des incompréhensions ${ }^{3}$.

11 Pour Angenot, ces "dialogues de sourds " sont insurmontables plutôt que profonds parce que - dans des termes qui font écho à l'article de Fogelin (1985) sur les désaccords profonds -

les règles mêmes de l'argumentation et les présupposés fondamentaux quant à ce qui est "rationnel », « évident », « démontrable », « connaissable » ne forment pas ou ne forment plus un terrain commun... les adversaires d'idées finissent par se percevoir les uns les autres comme des «fous" et renoncent tout simplement et fort raisonnablement à discuter entre eux (Angenot 2008 : 16).

C'est le caractère raisonnable invoqué ici qui devrait retenir notre attention. Le jugement n'est pas unilatéral, il est réciproque : les adversaires se perçoivent tous deux comme irrationnels et considèrent qu'ils sont raisonnables dans leur refus de se parler.

13 En fin de compte, on peut reformuler la question en termes philosophiques, en se référant à la façon dont Grice comprend la signification (et sa reconnaissance). Qu'estce que cela signifie pour un locuteur (ou un énonciateur) de signifier quelque chose? 
Grice propose un nombre de formulations changeantes, mais l'essentiel de ce qu'il entend est résumé dans la citation suivante :

« $E$ (énonciateur) voulait signifier quelque chose en énonçant $x$ » est vrai si, pour un auditoire donné, $E$ a énoncé $x$ avec l'intention: (1) que $A$ produise une réponse particulière $r$. (2) que A pense (reconnaisse) que $E$ voulait (1). (3) que $A$ accomplisse (1) sur la base de son activation de (2) (Grice 1969 : 92)

C'est un point de vue qui se focalise sur l'auditoire : dans ce cadre la signification, pour accéder à l'existence, nécessite une réaction de l'auditoire. Le pas décisif est le second : il faut que l'auditoire reconnaisse l'intention, de la part du locuteur, de signifier quelque chose. Dans les versions les plus extrêmes, quand les positions s'avèrent totalement incommensurables, le problème n'est pas que chacune des parties ne comprend pas ce que l'autre veut dire : c'est qu'elle se refuse à reconnaître que ce que l'autre dit fait sens. Richard Weaver représente une position intransigeante de ce type lorsqu'il soutient qu'il n'est pas possible de discuter avec des communistes : "Vous ne pouvez pas parler avec quelqu'un dont les prémisses de base sont totalement incompatibles avec les vôtres. Les mots prononcés sont dénués de sens, si bien qu'en réalité il n'y pas eu de discours " (1978: 557 ; je souligne). Et l'anthropologue Clifford Geertz donne un exemple graphique de ce qui est en jeu dans son analyse détaillée des combats de coqs au Bali et de leur rôle par rapport à l'image publique des participants. Comme l'explique Geertz, on peut adopter deux perspectives sur le même phénomène. Dans une analyse utilitaire à la manière de Bentham, un tel engagement dans le jeu devient tellement risqué qu'il paraît irrationnel de s'y lancer ; le fait de mettre en jeu sa persona ne fait qu'accroître cette irrationnalité. D'un autre côté, pour les habitants de Bali, «ce que cela renforce, c'est le caractère signifiant du tout» (what it mainly increases is the meaningfulness of it all - Geertz 1973 : 434). Les gens trouvent du sens dans des choses fort différentes; et ceux de Bali jugent rationnel ce que l'utilitariste (à la manière de Geertz) trouve irrationnel.

Pour répondre aux considérations émises par Angenot, Weaver et Geertz, et en suivant Grice, la reconnaissance de la signification doit être placée au sommet de la liste des conditions qui rendent l'argumentation possible. L'exigence de Grice pourrait être la plus rigoureuse, et il faut se demander si elle n'est pas trop stricte. Elle est liée à la reconnaissance des intentions de l'autre, ce qui impliquerait déjà en soi l'existence d'une trame de significations sous-jacente! Le sens d'un énoncé présuppose la profondeur d'une expérience ancrée dans les significations sociales. Je peux certes reconnaitre une intention de signifier quelque chose, mais Grice exige que je reconnaisse l'intention de susciter la réponse $r$ à la suite d'une interprétation correcte de l'énoncé $x$, et ceci dépasse mes capacités si je me situe à l'extérieur de la société en question. Il y a une différence entre les comportements humains et le sens social qu'on leur attribue. S'inspirant de Gilbert Ryle (1968), Geertz (1973) juxtapose la description "dense» ou "épaisse» (thick description) et ce qu'il appelle thin description, la description «mince » et présente l'ethnographie comme une sorte d'effort intellectuel tourné vers la description épaisse (Geertz 1973:5-6). L'exemple paradigmatique favori de Ryle et de Geertz est le clin d'œil. Ici, la simple action de contracter la paupière possède des significations sociales. Une description mince se contente de mentionner la rapide contraction de la paupière, un simple spasme musculaire. Par contre, une description épaisse se réfère à un code social pour expliquer cet acte comme établissant une connivence entre la personne qui cligne de l'œil et son auditoire. En réalité, c'est dans la reconnaissance que l'échange relève de la connivence que le clin d'œil se donne 
comme " un clin d'œil». Contrairement au spasme musculaire de la paupière, le clin d'œil est délibéré, intentionnel. Ainsi, le recoupement des codes sociaux informe le comportement, qui à son tour peut être traduit en énoncés. Les codes sociaux - et les codes rhétoriques d'Angenot - sous-tendent des comportements intentionnels.

En conséquence, pour explorer ce problème tel qu'il a été transmis par ces penseurs, nous pourrions nous tourner vers des cas où la difficulté a dû être surmontée ; des cas où le discours et le comportement n'ont pas été reconnus comme produisant du sens. Ma suggestion est que des incursions dans ce qu'on peut appeler une anthropologie de l'argumentation peuvent être une bonne façon d'accomplir cette tâche. On peut déjà tirer de la notion de description épaisse et de l'exemple du clin d'œil, qu'un véritable échange signifiant peut être défini précisément de cette façon - c'est un événement social imprégné de signification pour tous ceux qui y sont impliqués. À divers moments de l'histoire de l'humanité (généralement, mais pas toujours, aux moments de la conquête européenne) des communautés différentes se sont trouvées en contact pour la première fois. Ces moments de contact, dans la mesure où ils peuvent être reconstitués, offrent des exemples précieux des conditions qui précèdent toute argumentation entre communautés. Ils servent ainsi de leçons pour comprendre les cas contemporains où semble se faire jour un conflit de rationalités. Et ils nous permettent de sortir de l'impasse dans laquelle la position d'Angenot nous enferme.

\section{Rhétorique de la rencontre}

17 J'utilise délibérément le concept de «rhétorique de la rencontre ». Il ne manque pas de soulever des problèmes en raison du flou de la notion de « rencontre ». Cependant, en l'absence d'une meilleure alternative, il fournit un espace conceptuel qui peut être investi avec profit pour dévoiler un ensemble d'idées associées aux premières interactions entre des cultures étrangères l'une à l'autre. Telle que je l'entends, la "rhétorique de la rencontre" consiste dans la juxtaposition de systèmes de significations rhétoriques mis en contact de façon originale et dans l'espoir (sinon l'objectif) d'établir un environnement cognitif partagé.

18 Cette définition implique différents éléments, dont les plus importants sont: (1) que les systèmes rhétoriques (ce qu'Angenot appelle des codes) participent d'une expérience humaine universelle. Nous sommes des êtres rhétoriques, ce qui veut dire que nous utilisons le discours et d'autres stratégies pour modifier la réalité sociale et que nous avons une capacité d'écoute, et sommes ouverts à ce type de modifications. Chaque communauté ou société développe ce type de système rhétorique, que ce soit sur le mode de l'isolement ou sur celui de l'échange avec d'autres communautés. (2) Deuxièmement, des modifications de cet ordre ne surgissent pas nécessairement à la suite d'échanges directs entre individus; elles peuvent survenir à la suite de changements dans l'environnement cognitif au sein duquel les gens agissent, et où les significations sont construites et partagées. Comme ces environnements se chevauchent, nous avons affaire à des environnements cognitifs mutuels. Les modifications indirectes des points de vue ne correspondent pas à une lecture stricte de la façon dont Grice rend compte de la signification; elles accordent plus de place au rôle des auditeurs comme récepteurs actifs de la signification. Ils se situent aussi endehors des irrémédiables « ruptures cognitives » présentées par Angenot. En fait, c'est 
grâce à des environnements cognitifs mutuels que de telles coupures peuvent être surmontées.

Pour donner une idée de ce qui se joue dans les premiers contacts entre peuples, je me réfère au travail de l'historienne australienne Inga Clendinnen. Elle a longuement réfléchi aux questions qui me préoccupent ici. Un éloge funèbre dans The Australian la décrit comme «fascinée par la logique et la consistance du contact entre des peuples qui s'ignoraient totalement ${ }^{4} »$. C'est dans cette perspective qu'elle apparait comme une source, et une voix importante dans le présent débat. Mais cette voix fait entendre une mise en garde. Parlant des Aztèques, par exemple, elle note : "Je savais qu'ils étaient humains, et j'en avais un rappel une douzaine de fois par jour. Mais il est vite devenu évident que leur esprit et leurs émotions étaient différents des miens » (2006: 21-2). Et elle continue :

Cela seul me retenait de leur appliquer de façon commode notre alphabet simplifié d'émotions - «la peur », «la pitié », «la colère ». Le cocon de sécurité physique dans lequel nous vivons pourrait être le plus grand obstacle à la compréhension de la façon dont les choses se passaient pour d'autres gens en d'autres temps, ou pour comprendre comment elles se passent pour des gens qui vivent ailleurs de nos jours. Ce changement radical des conditions de vie fait de tout espoir d'« empathie» une fiction (2006: 26 , je souligne).

Qu'est-ce que cela pourrait signifier pour des façons de penser et de sentir d'être sujets à un autre ordonnancement? Car nous revenons ici à l'opposition entre raison/ émotion invoquée par Hare, et même si nous adoptons une vision de la cognition qui nous permet de parler de cohérence émotionnelle (Thagard 2012), c'est la différence entre la combinaison de l'expérience et du sentiment chez les uns et les autres que Clendinnen relève. Rien de ce qu'on avait compris jusque-là de l'accès à la signification n'est traité de façon claire par les remarques de Clendinnen sur les Aztèques. Car ici elle ne parle pas d'une rencontre entre deux cultures anciennes, mais de sa rencontre en tant qu'historienne avec des sujets situés dans le passé. Bien que cela même puisse être une différence de signification, sa rencontre s'effectue au niveau d'un examen minutieux de type abstrait ; elle n'est pas immergée dans une instantanéité où elle doit traiter l'altérité selon les modalités que nécessite une interaction avec les autres, parce qu'il n'y a pas d'interaction. Elle apporte une vision perspicace et l'expertise de l'historienne, les vraies rencontres de première main se trouvent dans son matériau source.

21 Clendinnen apporte deux études instructives de rhétorique de la rencontre entre différents continents. Dans chacune d'entre elles, nous sommes témoins de la stupéfaction des peuples qui s'efforcent de comprendre ce que l'autre veut dire. Elles décrivent des expériences rhétoriques très différentes.

Dans Dancing with Strangers (2005), elle rend compte de la rencontre qui a eu lieu en 1788 entre la Première Flotte Britannique et les peuples de ce qu'on appellera plus tard l'Australie (elle les nomme Australiens). Dès l'abord, elle pose des limites :

A mon avis le monde sacré des Australiens en 1788 - le monde intellectuel et spirituel, dont rien n'est consigné par écrit mais qui est présent dans le paysage, les artefacts, la dance et les récits - est fermé à toute personne extérieure. Mon intérêt se porte en conséquence sur la vie séculière des Australiens: sur ce que nous pouvons apprendre des observateurs britanniques pendant ces quelques premières années de contact $(2005: 5)$. 
Étant donné qu'une partie de la rencontre reste opaque, elle est obligée de se fier aux voix et aux réactions de l'autre, qui lui-même est séparé d'elle par des couches de significations culturelles, certes reliées historiquement à « nos » (ou ses) significations, mais seulement dans le sens ancestral.

Cependant dans sa remarque, elle identifie aussi un élément du code rhétorique des Australiens. Quand elle parle $\mathrm{du}$ «monde intellectuel et spirituel, dont rien n'est consigné par écrit mais qui imprègne le paysage, les artefacts, la dance et les récits ", elle capte des détails de la façon dont ils stockaient et extrayaient de la signification une façon qui donne accès à un mode différent d'appréhension de l'univers.

De nombreux rapports de Clendinnen comprennent une interprétation des actions, qui est un instrument essentiel de l'ethnohistoire :

ce que nous appelons histoire quand les peuples qui suscitent notre curiosité n'ont pas laissé de documents déchiffrables, et quand leurs intentions et leurs interprétations doivent être reconstruites à partir des descriptions fournies par des étrangers lettrés qui souvent ne savent pas ce qu'ils regardent (un mariage? une célébration guerrière) ? (ibid. : $2005:$ 59)

Elle en viendra plus tard à parler d'un monde fait «d'une galerie de miroirs » (227), d'observation et de réflexion, et de construction d'explications sur ce qui est observé d'une façon aussi consistante que possible. Qu'on examine ce qui suit :

Une conversation est sur le point de débuter, il est donc bon de supputer quel est le probable degré d'exactitude de ce que nous allons entendre. Toute investigation de premières rencontres culturelles est entachée d'incertitudes concernant l'adéquation de la communication. Initialement, chaque parti peut seulement interpréter les actions et les intentions de l'autre selon son propre dictionnaire de gestes exprimés dans une sorte de pantomime (souvenez-vous du vocabulaire de signes de Morton ${ }^{5}$ ). La communication verbale est négligeable, et en général encore plus déficiente que ce que les locuteurs pleins d'espoir avaient pu supposer (ibid.: 112-113).

27 À cause du fait qu'ils dépendent des actions, les récits de Clendinnen sont invariablement des récits d'échec de la communication et d'erreurs de calcul. Imposant la loi et l'ordre, le Gouverneur britannique voyait « le bon ordre donné à contempler, avec Dieu et le Roi derrière le poteau de flagellation et le gibet ", tandis que les Australiens y voyaient une « cruauté répugnante et gratuite » (189). Et les Britanniques, qui accordaient une valeur suprême à l'honnêteté et aux serments, étaient choqués par la légèreté avec laquelle les Australiens niaient leur culpabilité même quand elle sautait aux yeux, parce que pour eux « le degré de culpabilité et les limites de la responsabilité dans un cas particulier étaient matière à négociations ou, si suffisamment graves, devaient être déterminés par une épreuve " ${ }^{6}($ ibid. : 194).

L'Histoire de Clendinnen est pleine de ce type de différences qui touchent au malentendu, un trait qui semble inévitable dans les rencontres culturelles du passé. Peut-être qu'un terme plus juste que « différences » serait ici « dichotomies » puisque, comme Geertz (1995 : 28) nous le rappelle, le premier met en jeu une comparaison qui relie; tandis que "dichotomies" se rapporte à des ruptures qui isolent. Ce qui en ressort est la façon dont ces moments de rencontre plongent les gens dans un état de stupéfaction devant l'étrangeté des comportements de l'autre.

29 Ces relations des rencontres culturelles du passé qui mettent la rhétorique au défi se reproduisent chaque fois qu'il y a confrontation avec ce qui ne nous est pas familier. 
30 Pas plus tard que dans les années 1960, le gouvernement australien a essayé de vider de ses habitants une région désertique de l'Australie de l'Ouest pour y faire des expériences nucléaires. La conséquence en fut que les aborigènes d'Australie sortirent du désert et pour la première fois rencontrèrent des blancs, et les pièges de la culture blanche. Lorsqu'ils relatent leur expérience après des dizaines d'années, nous entendons ceux qui se trouvent de l'autre côté de la relation parler de l'étrangeté avec laquelle ils se sont trouvés confrontés.

Nous avons vu deux hommes qui se tenaient debout. Ils avaient d'immenses chapeaux sur la tête. Nous pensions qu'ils portaient des plats, mais c'était seulement des chapeaux.

Ils nous ont mis à l'arrière d'une Toyota et c'était la première fois que nous avons roulé en voiture. C'était effrayant de voir tous les arbres, les herbes - qui couraient (toutes les citations sont reprises de Davenport, Johnson et Yuwali 2005).

31 Ces remarques, qui rappellent la poésie des Martiens à la mode en Grande-Bretagne dans les années 1970, sont clairement marquées par les décades pendant lesquelles les témoins ont vécu dans une autre culture. Ils savent maintenant ce qu'ils ont rencontré pour la première fois, comme les voitures par exemple. De ce fait, leurs témoignages ne reflètent pas pleinement l'altérité de l'expérience de la rencontre initiale. Néanmoins, ils rentrent dans le cadre de ce qui nous retient ici, et ils reflètent une expérience qui n'est pas ancrée dans un passé historique lointain.

\section{Les conditions préliminaires de l'argumentation}

32 Après avoir circonscrit le problème et l'avoir examiné plus avant, je tenterai maintenant de tirer de ce qui précède quelques suggestions sur les façons de comprendre les conditions préliminaires de l'argumentation.

33 L'une des choses à examiner en premier lieu est le sens à accorder au terme de "préliminaire » dans cette investigation. Qu'est-ce qui compte, et qu'est-ce qui ne compte pas? Il y a un sens général, évident, selon lequel ce dont il est question doit venir d'abord (précéder). Plus spécifiquement, ce qui est fait doit l'être en préparation de, ou par anticipation de, quelque chose d'autre. Dans ce qu'on peut considérer comme les circonstances normales de l'argumentation, les conditions préliminaires sont en place d'une façon qui les rend invisibles, tacites, si bien qu'elles n'attirent pas l'attention. Certains théoriciens voient dans l'accord une condition importante. Ainsi par exemple la pragma-dialectique conçoit l'argumentation comme une discussion critique qui procède en quatre stades, le premier desquels est appelé le stade d'ouverture (van Eemeren et Grootendorst 1984: 85-8) : les débattants établissent les règles qui vont gouverner leur discussion et se mettent d'accord sur les propositions qu'ils peuvent utiliser. Pour les pragma-dialecticiens, ces accords sont importants pour obtenir un résultat satisfaisant touchant au fond de la question discutée, et de ce fait, il convient dans une approche normative de les recommander pour assurer le bon développement de l'argumentation. Or, les circonstances de notre cas ne sont pas "normales» dans ce sens particulier. Les situations décrites dans les précédentes sections sembleraient exclure des accords de ce type parce que ces derniers présupposent des choses qui, en l'occurrence, ne sont pas encore en place. Dès lors la condition de la pragma-dialectique, bien que préliminaire, n'est pas de l'ordre de celles 
qui nous concernent; les moments d'une première rencontre ne se prêtent pas à des accords dynamiques entre agents « rationnels».

\subsection{Reconnaître un comportement significatif} que constitue le clin d'œil, nous pouvons ne pas comprendre ce que signifie quelque chose qui ne nous est pas familier; mais nous pouvons en général percevoir qu'il a une signification pour les autres, parfois même qu'il se propose de signifier quelque chose pour nous. Ceci nous ramène une fois de plus à la condition de reconnaissance formulée par Grice, une condition dont nous avons convenu précédemment qu'elle faisait défaut aux situations de première rencontre. Sans aucun doute, elle en est absente au sens plein du terme. Mais il y a dans notre expérience bien des éléments qui nous permettent de faire des inférences sur le comportement des autres, qu'il soit dirigé vers nous ou vers les membres internes d'un autre groupe. Nous pouvons juger que certaines choses ont une signification pour les membres d'un groupe par la manière dont ils lui accordent de l'attention, par la façon dont ils les mettent en relief, et les manifestent dans leurs actions.

Être interpellé dans des situations argumentatives est quelque chose qui nous est familier et ne nous cause aucun inconfort. Cela fait partie de notre expérience sociale, de notre être dans le monde. Et être interpellé c'est voir notre attention retenue. C'est bien ce que réclame l'argumentation. Nous l'expérimentons et, grâce à cette expérience, nous pouvons la reconnaître dans des circonstances moins familières qui touchent aux autres.

Philippe-Joseph Salazar (2015) explore différentes voies par lesquelles le non-familier sous la forme de l'État islamique s'intègre dans l'expérience de jeunes Français. L'une de ces voies est celle de l'esthétique. Dans le monde occidental, nous voyons tout particulièrement dans le logos (le logique) un mode de démonstration. Mais au niveau de la persuasion, qui toujours affleure dans ces discussions, nous inclurions l'ethos (le caractère) et le pathos (l'émotion), deux "preuves» dont Aristote a bien indiqué qu'elles opéraient avec une grande puissance dans l'argumentation sociale. Le Caliphat ajoute l'esthétique à cette triade, bien que nous puissions voir que celle-ci entretient un rapport avec la tradition occidentale du pathos. Les vidéos produites par le Caliphat, avec les bandes-audio de chant rythmique et les cadences en mesure des voix, attirent ceux qui les regardent et les écoutent, et qui comprennent rarement l'arabe. Salazar établit un parallèle avec le "plaisir " éprouvé par de nombreux participants à une messe tenue en latin, alors qu'ils ne comprennent pas le latin. Dans tous les cas, « ils perçoivent, ils sentent, ils sont dans la sensation, ce qui se nomme proprement une "attitude esthétique" » $2015: 126)$. Le passage au « sentir » est important, mais il n'est pas totalement étranger à la théorie de l'argumentation occidentale. Il rappelle quelques-uns des modes de l'argumentation multimodale mentionnés par Michael Gilbert (1997), en particulier le kiscéral. C'est le mode le plus abstrait des quatre dont traite Gilbert; il est tiré du japonais ki (pour énergie ou force vitale),et relève " de l'intuitif, de l'imaginatif, du religieux, du spirituel, et du mystique » (1997: 86). C'est donc le mode de communication qui s'alimente au religieux et au mystique. Le kiscéral, déclare Gilbert, « inclut de nombreuses sources d'information qui ne sont pas prises en compte dans la tradition rationaliste (ibid.: 88). Dans la même perspective, les

Argumentation et Analyse du Discours, 25 | 2020 
théoriciens de l'argumentation ont prêté une attention soutenue à l'aspect prosodique de certains arguments. La prosodie, qui est l'étude des mètres poétiques, s'intéresse à l'accentuation et aux formes d'intonation d'un énoncé. On peut juger de la façon dont elle se rapporte au chant en mesure des bandes-audio du Caliphat. Leo Groarke et Gabrijela Kišiček (2016) examinent la manière dont la prosodie peut être utilisée pour communiquer les prémisses et les conclusions d'un argument. Il s'agit là d'un travail relativement neuf, mais il se fonde sur la tradition qui considère que les arguments sont dirigés vers la personne tout entière, et non vers un être purement logique détaché de sa pleine humanité. Comme David Hume l'avait fait remarquer, certaines idées sont juste "ressenties » comme plus fortes et dans ce sens nous les enregistrons différemment que d'autres idées (Treatise, I, Part 3. Section 7). Selon Salazar, ce qui est en jeu c'est un drame comportant des enjeux politiques élevés qui « nous engage, pardelà la raison, dans le domaine de la sensation qui entraîne, élève et donne du sens » (2015: 127). De la sorte, nous reconnaissons la possibilité d'un échange signifiant là où il pouvait auparavant nous avoir échappé.

\subsection{Respecter l'altérité}

«L'altérité » est une vaste catégorie. Le terme se réfère non pas seulement à d'autres personnes, mais à leurs façons d'être. Le comprendre invite à se placer en imagination dans des contextes totalement non-familiers. C'est sans nul doute une tâche difficile. Le vieux dicton rhétorique qui conseille de se mettre dans les souliers de l'autre » est un défi quand - pour filer la métaphore - ces souliers ne conviennent tout simplement pas. Clendinnen distingue parmi ses correspondants-sources ceux dont les efforts conduisent à une appréciation croissante de la nature de l'autre, dans laquelle ils reconnaissent un autre "mode d'être ». Mais c'est là un phénomène qui doit se développer sur l'axe du temps, c'est une expérience qui demande une acclimatisation, comme toute tentative de passer d'une culture à une autre.

Dans un autre contexte, Clendinnen poursuit son questionnement sur ce qui est ici en jeu : «Avec le temps, un degré raisonnable de "compréhension" de tels gestes locaux deviendra possible, mais cela se fera lentement, dans la confusion et par tâtonnements, ce qui suppose de suivre un chemin sinueux fait d'observation, d'inférence et d'expérimentation » (1999: 89). Ici, c'est le mouvement vers l'altérité qui se donne comme un chemin ou une route faite "d'observation, d'inférence et d'expérimentation ». Ce sont ces activités cognitives qui permettent de progresser et qui transforment les contextes dans lesquels on évolue. Dans un monde où seul le local existe, où la quête de l'universel a été entravée au point que tout effort supplémentaire pour l'atteindre semble futile, l'invitation à de tels mouvements vers l'altérité est omniprésente. Si donc nous restons à l'intérieur du familier, c'est uniquement par choix, peut-être un choix fait d'obstination.

Nous sommes, dès lors, entourés de mystère: les façons d'être des autres sont énigmatiques. Pas seulement celles du musulman, ou du Mexicain, ou du marxiste, mais aussi du chimiste, du conservateur, et du conseiller juridique d'entreprise. Chacun organise sa vie de façon différente; chacun a sa façon de parler et ses modes de connaissance qui le séparent de ceux qui ne font pas partie du groupe. Même s'il y a de multiples points de recoupements, le noyau reste en grande partie opaque pour le noninitié ou l'ethnographe. 
Dans cette optique, on pourrait rappeler les remarques de Perelman et olbrechtsTyteca dans leur discussion sur la force des arguments. Au-delà de l'efficacité que les arguments peuvent avoir pour des auditoires particuliers, et leur capacité à résister aux objections, les arguments doivent répondre à des critères généraux de validité dans différents domaines de savoir. Comme ils l'expliquent (1970:616)

Toute initiation à un domaine rationnellement systématisé, non seulement fournit la connaissance des faits et des vérités de la branche en question, de sa terminologie propre, de la manière d'user des instruments dont elle dispose, mais éduque aussi dans l'appréciation de la force des arguments employés en cette matière.

41 Nous pouvons étendre le domaine de ces «branches" de savoir pour y inclure toute communauté qui se forme autour de vérités et de faits, et autour d'une terminologie spéciale, qui leur sont particuliers. Ce sont les communautés qui nous forment, qui définissent nos identités : ce sont elles qui mettent en place les critères du raisonnable qui informent notre façon de raisonner. En tant que telles, elles commandent le respect, ce même respect que nous exigeons à l'égard de nos propres communautés, et qui déjoue les préjugés dont se nourrissent les accusations d' «irrationalité» mentionnées dans l'ouverture de mon propos ${ }^{7}$.

\section{Un environnement cognitif mutuel}

42 Comme indiqué plus haut, l'un des indicateurs principaux de l'efficacité d'une argumentation est sa capacité à modifier l'environnement cognitif de l'auditoire. Cet environnement, Sperber et Wilson (1986) ont été les premiers à y voir un parallèle avec le champ visuel. En effet, comme dans le champ visuel, de nombreux éléments passent inaperçus, si bien que cognitivement, nous avons accès à plus qu'à ce que nous connaissons sur le mode actif. Il résulte de cette description que l'argumentation a besoin de s'accomplir dans un contexte d'environnements cognitifs mutuels, où la quantité de signification partagée est suffisante pour qu'on puisse donner et recevoir des raisons de façon adéquate. C'est pourquoi la définition de la «rhétorique de la rencontre " inclut la perspective d'établir un environnement cognitif mutuel. Aussi ardue qu'ait été cette tâche dans de nombreuses situations, et bien qu'elle émerge, pour faire une fois de plus écho à Clendinnen, «lentement, dans la confusion et par tâtonnements, ce qui suppose de suivre un chemin sinueux », c'est une condition préalable nécessaire au succès de l'argumentation.

Une fois de plus, les observations de Perelman et Olbrechts-Tyteca s'avèrent instructives : "Pour qu'il y ait argumentation, il faut que, à un moment donné, une communauté des esprits effective se réalise " $(1970: 18)$. Mais qu'est-ce qui accède au rang de "communauté des esprits effective"? Qu'est ce qui permet d'identifier une communauté ? L'existence d'un langage commun est certes indispensable. Mais un tel langage a comme contenu des significations partagées, des codes sociaux, qui rendent possible une véritable communication.

Dans les cas qu'explore Clendinnen, avec le temps, les environnements cognitifs des deux ensembles de participants ont été considérablement modifiés dans la mesure où une nouvelle façon d'être (de penser et d'agir) a été générée dans les esprits de part et d'autre. Dès lors qu'ils se sont mis à développer un fonds de significations commun, dont certaines neuves, et d'autres fondées sur les interprétations des significations de 
l'autre, un environnement cognitif mutuel a émergé. Voir comment un environnement cognitif mutuel en vient à se développer constituerait une tâche fascinante, mais très exigeante. Des cas de ce genre nécessiteraient en eux-mêmes des études à consigner dans des articles à part entière. Nous pouvons ici nous tourner vers le travail des ethnographes et des anthropologues qui ont pris part à ces processus dans leur démarche pour réaliser alors des études du non-familier (Goulet et Miller 2007). Mon seul but ici était de montrer la nécessité d'avoir des environnements cognitifs mutuels comme condition préliminaire de l'argumentation.

\section{Une compréhension élargie des « raisons »}

Hugo Mercier et Dan Sperber remarquent que « si les raisons n'étaient pas rationnelles à un degré suffisant, nous ne reconnaîtrions même pas leur statut de raisons " (2017: 139). Or, cette observation met en évidence la question même de savoir comment il se fait que nous reconnaissions comme telles des raisons, une question qui fait partie du débat exploré plus haut. Fish (1999) et Fogelin (1985) ont tous deux argué que des positions radicalement différentes échappaient à l'argumentation parce que ce qui compte comme preuve pour l'une des parties ne pouvait en rien être reconnu par l'autre. De ce fait, il n'y a aucune chance de trouver des prémisses communes susceptibles de servir de base, et à partir desquelles il serait possible d'évaluer chaque position de l'extérieur. Bien sûr, les positions peuvent être évaluées de l'intérieur, mais seulement dans les termes propres à ce même système de rationalité.

Une grande partie de ce que dont j'ai débattu dans cet article pourrait le confirmer. Parler de la nécessité d'être initié à des champs systématisés de façon rationnelle suggèrent que ces champs sont fermés aux non-initiés. Mais les conditions dans lesquelles nous avons convenu qu'ils peuvent être jugés rationnels côte à côte désigne une ouverture qui promet une possibilité d'accès. Les gens se déplacent effectivement entre différents champs, et d'autres, comme le journaliste scientifique, peuvent travailler à la périphérie des champs, transmettant leurs idées dans une communauté plus large de parties intéressées. Les systèmes fermés (comme ceux que nous avons examinés dans la rhétorique de la rencontre) ne peuvent rester fermés longtemps, dans la mesure où la curiosité naturelle et le désir de comprendre des expériences nouvelles va à l'encontre d'une telle clôture.

De plus, les exemples de la rhétorique de la rencontre permettent de saisir la variété des sources dont découlent les raisons qui peuvent initialement ne pas être reconnues comme telles dans un autre système rationnel. Le pas préliminaire nécessite néanmoins qu'ils soient reconnus comme étant bien des raisons pour ceux qui les considèrent comme telles, et ainsi que soient élargies les sources de preuve dont les raisons dérivent.

Ceci relève d'une autre exigence d'ouverture. C'est un défi à l'esprit occidental : on lui demande de considérer les rêves, les rituels, les paysages, et même les récits comme des sources de preuves légitimes qui forment des systèmes de connaissance et corroborent des vérités à l'intérieur de ces systèmes. Mais c'est précisément le défi à relever. Quand la cour de justice accepte un récit oral comme preuve des faits dans un cas de revendication territoriale, et lui accorde une valeur supérieure que celle qu'elle donne à des sources écrites, la catégorie des « raisons » s'en trouve élargie ${ }^{8}$. 
Percevoir quelque chose comme une raison nécessite des expériences communes ancrées dans un environnement cognitif mutuel. Mais reconnaître l'existence d'une raison en vertu du rôle qu'elle joue dans le raisonnement d'une autre communauté n'exige pas, au départ, de compréhension de cet ordre. La reconnaissance est le premier pas vers la compréhension, après laquelle vient l'acceptation. Si la rhétorique inclut la capacité de détecter les moyens de persuasion disponibles, alors l'argumentation rhétorique, comme partie de cette même capacité, inclut le fait de déceler des raisons.

\section{Conclusions}

50 Immergées dans le non-familier, les rencontres avec la différence, qu'elles se passent dans notre entourage ou au loin, sont le fond de toile sur lequel on interprète, communique et argumente avec succès. Comme le saisit bien le psychologue Jerome Bruner (1990: 95), il est inévitable que des sociétés différentes construisent le réel différemment. Dès lors, il est également nécessaire que la théorie de l'argumentation développe des instruments pour parvenir à tenir compte de cette diversité.

51 Tout théoricien qui se confronte à ce problème est dans l'obligation d'apporter une réponse susceptible de servir de remède à la situation. Historiquement, suggère Angenot, le problème disparaît en même temps que les adversaires en présence et l'apparition d'une nouvelle génération qui n'accorde plus guère d'importance à la nature du différend ou au sujet traité. Ce n'est évidemment pas là un avis suffisant attendez jusqu'à ce que la situation qui nous met dans une impasse se résolve d'ellemême - et ce n'était d'ailleurs pas l'intention de départ: il s'agit seulement d'une observation sur ce qui s'est dégagé de l'examen de quelques cas.

L'argumentation rhétorique va sans cesse au-delà du vérifiable, ce qui revient à dire que son domaine est celui de l'incertitude. Ceci a un impact sur la façon dont nous comprenons la «raison». Dans les manuels, elle est typiquement isolée de ses contextes, coupée des processus dynamiques dans lesquelles elle a opéré; c'est ce qu'Angenot appelle un mécanisme de recherche de la vérité désincarné et décontextualisé. Par contraste, nous nous intéressons à une raison qui est impliquée dans le monde: en d'autres termes, une raison qui est à la fois située et incarnée. Lorsque Richard Weaver écrit que «la rhétorique vient toujours à nous dans la chair des mots» (1970), il rend bien cette idée. Tandis que nous accédons à une activité « humaine », qui reflète l'humain et est réfléchie par lui, l'« humain » lui-même se perd dans la diversité des apparences. L'argumentation a réuni avec succès des parcelles de diversité, et a permis la gestion des désaccords que réclame la coexistence. Mais dans les cas particuliers, l'argumentation, comme je l'ai suggéré, ne s'élance jamais d'ellemême vers le devant de la scène. Le terrain doit être préparé, les conditions préliminaires mises en place. Les différentes discussions présentées dans cet article, avec les exemples analysés, ont esquissé les conditions qui doivent faire partie d'un ensemble plausible de "préliminaires». (1) Les leçons tirées du champ de l'anthropologie culturelle, et en particulier les «descriptions épaisses" qui soustendent les interactions sociales manifestent la nécessité d'identifier ce qui est significatif aux yeux des autres ; (2) la présentation de la rhétorique des rencontres, et plus particulièrement de la façon dont ce concept est illustré dans le travail de Inga Clendinnen, souligne l'importance d'établir une relation de respect envers « l'altérité » 
et de manifester une attitude d'ouverture à son égard ; (3) l'examen d'idées empruntées à Fish et Fogelin, et plus particulièrement à Angenot, indique que l'existence d'un environnement cognitif mutuel est impérative. Il est clair que certains environnements cognitifs sont plus riches que d'autres, comme le sont les différences qui existent entre eux. Mais la compréhension peut transcender ces différences, ce que montrerait un examen plus poussé des conceptions de ces théoriciens. (4) Finalement, l'attention portée à la rhétorique de la rencontre désigne un potentiel : celui de découvrir des raisons dans une panoplie de sources, et sous des aspects différents. Des cultures contrastées dotées d'une épistémologie qui diffère entre elles laissent entrevoir cette promesse, comme le montre dans l'œuvre plus contemporaine de Chaim Perelman et Lucie Olbrechts-Tyteca l'idée que les raisons dépendent des domaines dans lesquels elles s'inscrivent.

C'est là un ensemble inclusif de conditions; elles doivent toutes être présentes sous une forme ou une autre pour qu'une argumentation réussie soit possible. Il se peut que ce ne soit pas l'ensemble que prévoyaient Perelman et Olbrechts-Tyteca; mais il se conforme aux perspectives qu'ils ont ouvertes, et répond bien au défi qu'ils ont lancé 9 .

\section{BIBLIOGRAPHY}

Angenot, Marc. 2008. Dialogues de sourds. Traité de rhétorique antilogique (Paris : Mille et une nuit, collection Essais)

Billig, Michael. 1991. Ideology and Opinions (London: Sage)

Booth, Wayne C. 2004. « My Life with Rhetoric: From Neglect to Obsession », Jost, W. \& W. Olmstead (eds). Rhetoric and Rhetorical Criticism. (Oxford: Blackwell), 494-504

Bruner, Jerome. 1990. Acts of Meaning (Cambridge: Harvard U. P.)

Clendinnen, Inga. 2006. Quarterly Essay 23: The History Question : Who Owns the Past? (Melbourne: Black Inc.)

Clendinnen, Inga. 2005. Dancing with Strangers: The True History of the Meeting of the British First Fleet and the Aboriginal Australians, 1788 ( Edinburgh: Canongate Books)

Clendinnen, Inga. 1999. Reading the Holocaust (Cambridge : Cambridge U. P.)

Davenport, S., P. Johnson \& Yuwali. 2005. Cleared Out: First Contact in the Western Desert (Canberra: Aboriginal Studies Press)

Fish, Stanley. (1999) « Why We Can’t All Just Get Along, » The Trouble with Principle. (Harvard U. P.), 243-62

Fogelin, Robert. 1985. «The Logic of Deep Disagreements », Informal Logic 7:1, 1-8

Geertz, Clifford. 2000. Available Light: Anthropological Reflections on Philosophical Topics (Oxford: Oxford U. P.)

Geertz, Clifford. 1995. After the Fact: Two Countries, Four Decades, One Anthropologist. (Cambridge, MA: Harvard U. P.) 
Geertz, Clifford. 1973. The Interpretation of Cultures: Selected Essays by Clifford Geertz (New York: Basic Books)

Gilbert, Michael. 1997. Coalescent Argumentation (Mahwah, NJ: Lawrence Erlbaum)

Gómez, Carlos. 2012. Interculturality, Rationality and Dialogue: In Search for Intercultural Argumentative Criteria for Latin AmericaI (Würzburg : Echter Verlag)

Goulet, Jean-Guy \& Bruce Miller (eds). 2007. Extraordinary Anthropology: Transformation in the Field (Lincoln, NB: U. of Nebraska P.)

Grice, Herbert Paul. 1989. « Utterer's Meaning and Intentions, in Studies in the Way of Words (Harvard U. P.), 86-116

Groarke, Leo \& Gabrijel Kišiček. 2016. « Compassion, Authority, and Baby Talk : Prosody and Objectivity », Bondy, P. \& L. Benacquista (eds). Argumentation, Objectivity, and Bias: Proceedings of the 11th International Conference of the Ontario Society for the Study of Argumentation (OSSA), 18-21 May 2016. Windsor, ON: OSSA, 1-13

Hample, Dale. 2018. Interpersonal Arguing (New York: P. Lang)

Hare, Richard Mervin. 1970. « On Terrorism », The Journal of Value Inquiry, 241-49

Harris, Randy (ed.). 2005. Rhetoric and Incommensurability (Lafayette, IN: Palor Press)

Hume, David. 2000. A Treatise on Human Nature, Norton, D. Fate \& M. Norton (eds) (Oxford: Oxford U. P.)

Kock, Christian. 2007. « Norms of Legitimate Dissensus », Informal Logic 27:2, 179-196

Kraus, Manfred. 2012. «Cultural Diversity, Cognitive Breaks, and Deep Disagreement: Polemic Argument », van Eemeren, Frans H. \& Bart Garssen (eds). Topical Themes in Argumentation Theory (New York: Springer) 91-107

Mercier, Hugo \& Dan Sperber. 2011. «Why do Humans Reason? Arguments for an Argumentative Theory ", Behavioral and Brain Sciences 34, 57-111

Mercier, Hugo \& Dan Sperber. 2017. The Enigma of Reason (Cambridge, MA : Harvard U. P.)

Mill, John Stuart. 1999. On Liberty. E. Alexander (ed.) (Peterborough, ON: Broadview Press)

Perelman, Chaim \& Lucie Olbrechts-Tyteca. 1970 [1958]. Traité de l'argumentation. La nouvelle rhétorique (Bruxelles : Éditions de l’U. de Bruxelles)

Richards, Ivor Amstrong. 1936. The Philosophy of Rhetoric (Oxford: Oxford U. P.)

Ryle, Gilbert. 1968. The Thinking of Thoughts, or What is Le Penseur Doing? (University of Saskatchewan: University Lectures, No. 18)

Salazar, Philippe-Joseph. 2015. Paroles armées: Comprendre et combattre la propaganda terroriste (Paris : Lemieux)

Sperber, Dan \& Deirdre Wilson. 1986. Relevance: Communication and Cognition (Cambridge, MA: Harvard U. P.)

Thagard, Paul. 2012. The Cognitive Science of Science: Explanation, Discovery, and Conceptual Change (Cambridge, MA: MIT Press)

Tindale, Christopher. W. 2013. « Rhetorical Argumentation and the Nature of Audience: Toward an Understanding of Audience-Issues in Argumentation ", Philosophy and Rhetoric 46:4, 508-532 
Tindale, Christopher. W. 2016. « On Cognitive Environments », Mohammed, Dima \& Marcin Lewinski (eds). Argumentation and Reasoned Action. Vol. 1 (College Publications). 687-705

Tindale, Christopher.W. 2015. The Philosophy of Argument and Audience Reception (Cambridge: Cambridge U. P.)

Tsilhqot'in Nation v. British Columbia, 2014 SCC 44

Van Eemeren, Frans H. \& Rob Grootendorst. 1984. Speech acts in argumentative discussions: A theoretical model for the analysis of discussions directed towards solving conflicts of opinion (Dordrecht: Floris)

Weaver, Richard. 1970. « Language is Sermonic », Johannesen, Richard L., Rennard Strickland, \& Ralph T. Eubanks (eds). Language is Sermonic: Richard M. Weaver on the Nature of Rhetoric. (Baton Rouge: Louisiana State U. P.), 201-225

Weaver, Richard. 1978. « The Strategy of Words », Goodnight, Gerald Thomas. Rhetoric and Culture: A Critical Edition of Richard M. Weaver's Unpublished Works. Diss. U. of Kansas

\section{NOTES}

1. Le fanatique, déclare Hare, laisse à la place «libre cours aux émotions » (1970:248). De la sorte, Hare coupe totalement l'émotion de la raison, ce que refuseraient de faire de nombreux auteurs contemporains.

2. Sans doute, à un stade préliminaire à l'argumentation, le choix peut être encouragé si on met en lumière les alternatives qui existent dans le contexte où l'individu prend son engagement. J'ai appelé ceci ailleurs " un stade pré-argumentatif de la persuasion » (Tindale 2013: 527) où les possibilités de réceptivité sont renforcées. Mais le type de problème que je traite ici se pose préalablement à toute connaissance possible des engagements d'un individu (ou d'un groupe).

3. Ceci fait écho à l'approche de I. A. Richards envers la rhétorique, où la persuasion est seulement l'un des buts du discours et la définition essentielle de l'entreprise est celle d'une « étude de l'incompréhension mutuelle et de ses remèdes » (1936:3).

4. http://www.theaustralian.com.au/arts/books/inga-clendinnen-writing-for-her-life/newsstory/a8a881af697e09588ee98f260687afb7

5. Le Comte de Morton, Président de la Société Royale, avait en 1768 présenté au Capitaine James Cook l'alphabet rudimentaire d'un langage par signes dont il pensait qu'il ne pouvait être erroné (par exemple, montrer sa bouche et mâcher).

6. Ceci est un exemple de différence interculturelle. Les lecteurs noteront les parallèles avec des cas de différence intraculturelle comme ceux qui relèvent de la politique des USA sous la présidence de Trump.

7. Le concept est exploré de façon beaucoup plus approfondie, et sa pertinence pour la théorie de l'argumentation est exposée dans Tindale $(2015 ; 2016)$.

8. Ceci était un jugement de la Cour Suprême de la Colombie Britannique (2007), respecté par la Cour Suprême du Canada (2014) :Tsilhqot'in Nation v. British Columbia, 2014 SCC 44.

9. Je voudrais exprimer ma reconnaissance aux évaluateurs anonymes de la revue dont les commentaires attentifs ont permis un nombre de clarifications et d'améliorations dans ce travail. 


\section{ABSTRACTS}

Early in The New Rhetoric, Perelman and Olbrechts-Tyteca raise the spectre of the fanatic who "adheres to a disputed thesis for which no unquestionable proof can be furnished," refuses to submit it for free discussion, and thereby "rejects the preliminary conditions which would make it possible to engage in argumentation" (1970: 62). One of the many interesting suggestions here is that there may be preliminary conditions that should be in place before argumentation can be engaged. In this paper, I pursue that suggestion and consider what such preliminary conditions should be, in order to address problems of dialogue between people or groups who seem to subscribe to different conceptions of rationality. I draw from some examples of confrontations between peoples for the first time (encounter rhetorics), where those involved had first to come to understand each other before argumentation between them could develop.

Dans la Nouvelle rhétorique, Perelman et Olbrechts-Tyteca évoquent d'entrée de jeu le spectre du fanatique qui «adhérant à une thèse contestée, et dont la preuve indiscutable ne peut être fournie, refuse néanmoins la possibilité de la soumettre à une libre discussion, et par conséquent, refuse les conditions préalables qui permettraient, sur ce point, l'exercice de l'argumentation » (1970 : 82). L'une des suggestions intéressantes de ce passage est qu'il peut y avoir des conditions préliminaires à mettre en place pour permettre à l'argumentation d'émerger. Dans cet article, je développe cette suggestion et tente de voir ce que pourraient être ces conditions préliminaires, dans le but d'examiner les problèmes qui se posent dans le dialogue entre des individus ou des groupes qui semblent souscrire à des conceptions différentes de la rationalité. Je m'appuie sur des exemples de confrontation entre peuples qui se rencontrent pour la première fois (rhétorique de la rencontre), dans lesquels les intéressés doivent avant tout parvenir à se comprendre avant qu'une argumentation entre eux puisse se développer.

\section{INDEX}

Mots-clés: altérité, argumentation interculturelle, environnement cognitif mutuel, raisons, rhétorique de la rencontre

Keywords: encounter rhetoric, intercultural argumentation, mutual cognitive environment, otherness, Perelman, reasons

\section{AUTHORS}

\section{CHRISTOPHER W. TINDALE}

University of Windsor 